Ann. Abeille, I966, 9 (I), 7I-76.

NOTE TECHNIQUE

\title{
NOUVEAU MODÈLE DE CAGETTES EXPÉRIMENTALES POUR LE MAINTIEN D'ABEILLES EN CAPTIVITÉ
}

\author{
Janine PAIN \\ Station de Recherches sur l'Abeille et les Insectes sociaux, \\ 91 - Bures-sur-Yvette
}

SOMMAIRE

L'auteur décrit un modèle de cagette en Altuglas ( ${ }^{1}$ ), spécialement conçue pour le maintien en captivité de petits groupes d'ouvrières. Il met en évidence le maniement aisé et le nettoyage facile de ces cagettes, particulièrement adaptées ì l'étude de l'alimentation des abeilles.

La cagette expérimentale décrite ci-dessous a été mise au point pour le maintien en captivité de petits lots d'abeilles dans des conditions répondant à un certain nombre d'exigences expérimentales. Elle a été entièrement réalisée par l'atelier de la Station de Recherches sur 1'Abeille et les Insectes sociaux ( $\left.{ }^{2}\right)$. Elle a été étudiée spécialement en vue des recherches sur l'alimentation des abeilles, mais peut servir également aux recherches sur le comportement des ouvrières : réactions aux stimuli spécifiques provenant de la reine, comportement de construction, etc.

Ce nouveau modèle s'inspire de celui de la Station de Liebefeld en Suisse, mais les dimensions en sont très différentes ainsi que la matière employée pour sa construction.

(1) Polyméthacrylate de méthyle (Altuglas ou Plexiglas).

(2) Nous remercions M. Charles, responsable de l'atelier, qui en a assuré l'exécution et qui a mis au point les méthodes de fabrication en petites séries.

Pain J., I $96 \mathrm{I}$. Sur la phérormone des reines d'abeilles et ses effets physiologiques. Thèse Sci. nat., Paris, $n^{\circ} 4526$. 


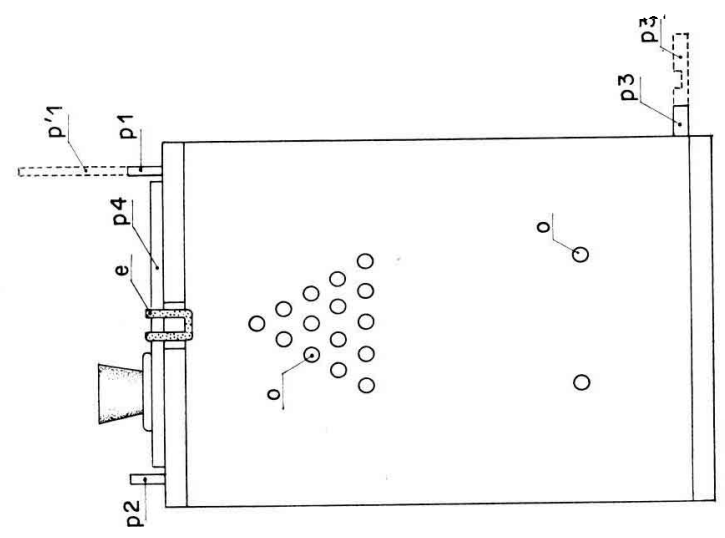

(
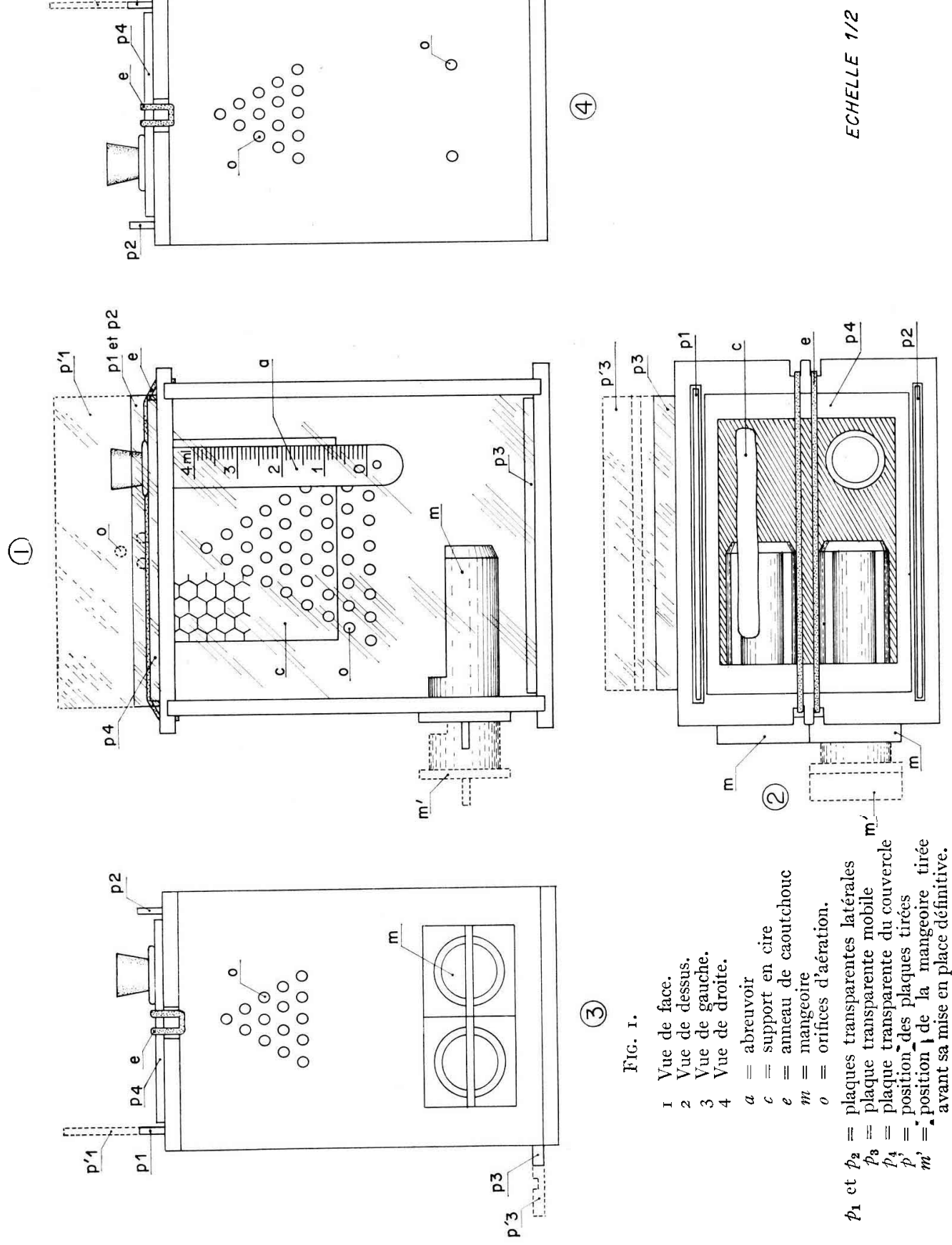


\section{DESCRIPTION}

La forme générale est un parallélépipède rectangle de II $\mathrm{cm}$ de haut sur $9,5 \mathrm{~cm}$ de large et $7,5 \mathrm{~cm}$ de profondeur.

Nous avons employé 1'Altuglas (polyméthacrylate de méthyle). Le socle et les deux petits côtés sont blanc opaque. Par contre, les deux grands côtés sont transparents, ce qui leur permet de faire office de vitres. Le dessus, qui forme toit, déborde de $3 \mathrm{~mm}$ environ sur les petits côtés. Il est formé de deux parties : un entourage en Altuglas blanc opaque ajouré au centre sur une surface de $7 \times 5 \mathrm{~cm}$ et sur lequel repose un couvercle transparent de $8,5 \times 5,8 \mathrm{~cm}$ maintenu par les deux côtés transparents. Ceux-ci, de dimensions II $\times 8,8 \mathrm{~cm}$, sont percés d'orifices d'aération de $3,5 \mathrm{~mm}$ de diamètre disposés en triangle. Ils peuvent être retirés facilement car ils glissent dans des rainures pratiquées dans les plaques opaques des deux petits côtés ainsi que dans deux fentes entaillées dans l'entourage blanc opaque du toit.

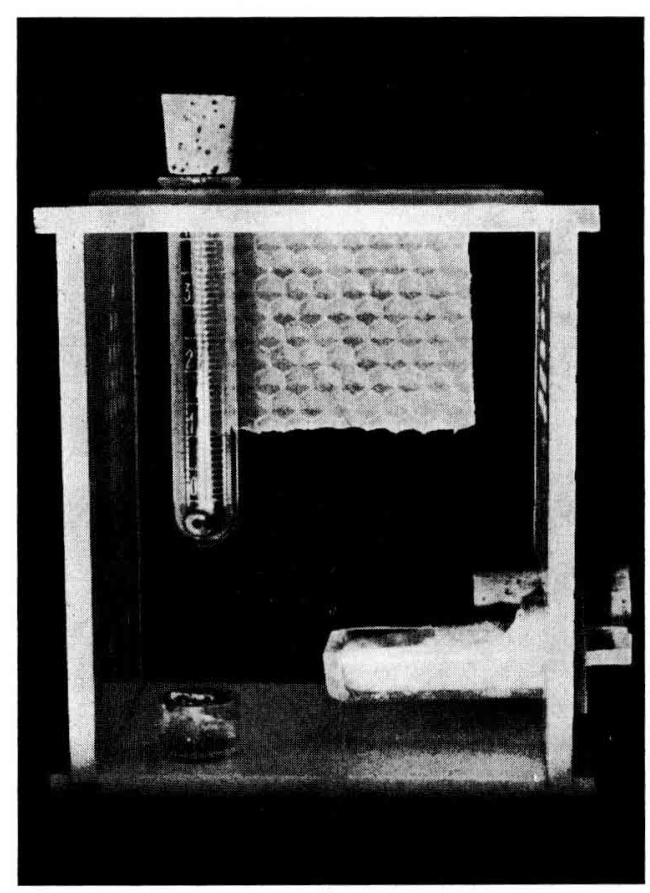

FIG. 2. - Modèle de cagelte monté

(photo B. David)

Le convercle transparent $d u$ toit peut être garni d'un support en cire : feuille gâufrée ou morceau de rayon. Il possède un orifice de $\mathrm{I}, 4 \mathrm{~cm}$ de diamètre dans lequel on glisse un abreuvoir en pyrex d'une capacité de $4 \mathrm{ml}$. Cet abreuvoir est percé à l'une de ses extrémités pour permettre aux abeilles d'absorber le liquide. Il est fermé à l'autre extrémité par un bouchon de liège. 


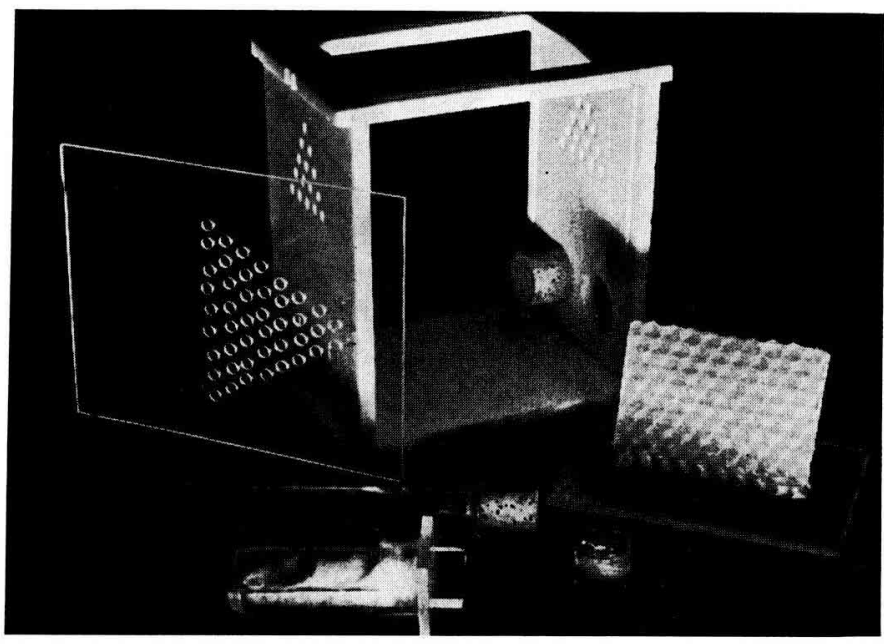

FIG. 3. - Moldele de cagette démonté

(photo B. David)

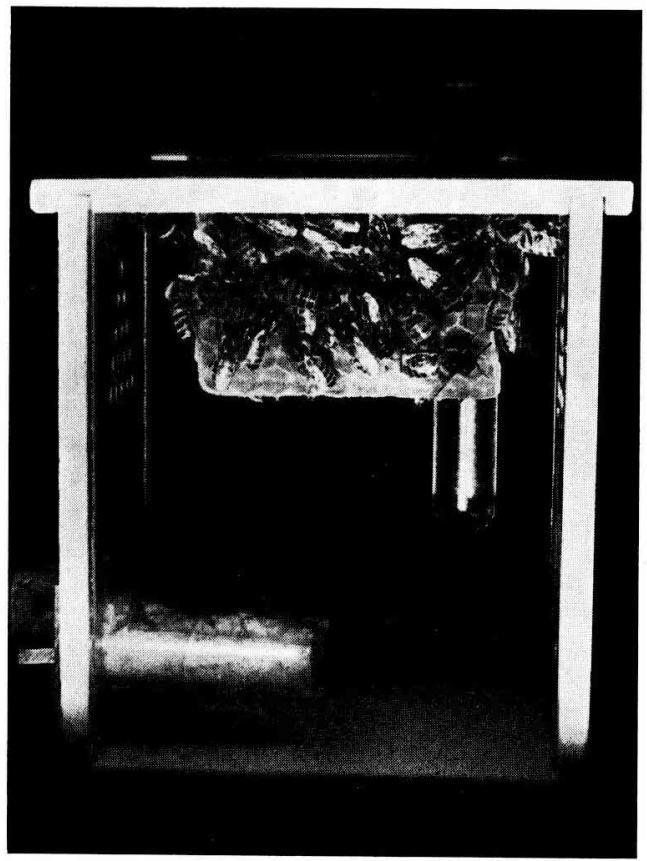

FIG. 4. - Cagette peuplée par une petite colonie d'ouvrières 
Les plaques formant les petits côtés sont perforées, comme les parois transparentes, d'orifices d'aération de mêmes dimensions mais disposés en un triangle moins important.

Sur l'un de ces petits côtés, deux perforations de $2 \mathrm{~cm}$ de diamètre ont été ménagées afin de recevoir deux mangeoires contenant l'une la nourriture sucrée (candi), l'autre la nourriture azotée (pollen) (fig. I à 6).

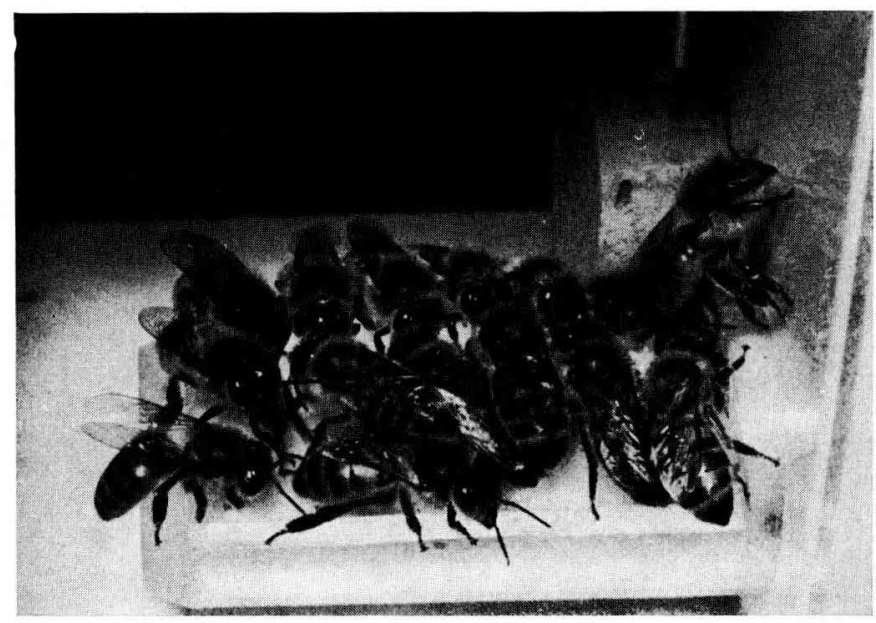

Firi. 5. - Groupe d'oubrières sur une mungeoire conlendun du sucre candi (photo B. David)

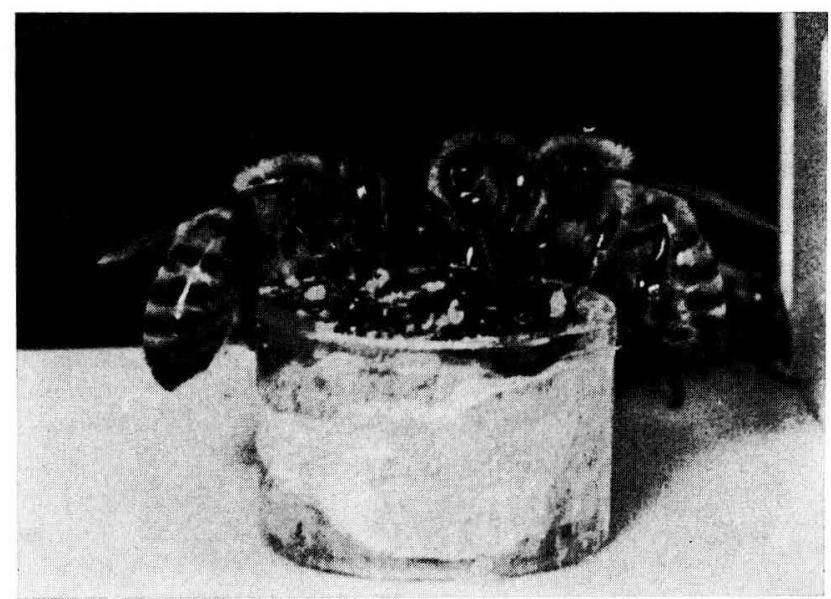

FI(i. 6. - Ouvrières consommant du pollen

(photo B. David)

Sur le socle de la cagette de dimensions $10,5 \times 5,7 \mathrm{~cm}$, on dispose intérieurement une plaque mobile transparente de $7 \times 8,3 \mathrm{~cm}$ sur laquelle a été creusée, sur la plus grande longueur, une rainure dans laquelle vient s'emboîter et se bloquer l'une des parois transparentes fonctionnant comme vitre. 
Ce modèle de cagette permet d'introduire les abeilles soit par l'une des perforations oì s'encastrent les mangeoires, soit par l'orifice réservé à l'abreuvoir. Deux cents abeilles peuvent y séjourner sans difficulté.

La nourriture est facilement administrée dans les mangeoires que l'on met en place à la façon de tiroirs. Chacune d'elles peut contenir Io $g$ de candi ou tout autre aliment pâteux ou solide. Elles sont d'une manipulation aisée et on peut les peser facilement car leur forme leur permet de tenir en équilibre sur le plateau d'une balance.

Des mangeoires supplémentaires de formes variées peuvent être encore introduites sur le fond de la cagette.

La ventilation est suffisante. Le nettoyage est facile. On peut changer aisément les deux parois transparentes ainsi que la plaque mobile posée sur le socle.

Ce modèle a l'avantage sur les anciennes cagettes en bois de ne pas retenir les odeurs qui peuvent être gênantes, surtout quand il s'agit d'étudier celles qui sont émises par la reine (PAIN, I96I).

La plaque transparente du couvercle assure un contrôle méthodique de la population encagée.

Il est possible, si la nécessité s'en fait sentir, de ménager deux nouveaux orifices dans le couvercle pour introduire des abreuvoirs supplémentaires.

On peut aussi coller à chaud sur le couvercle, deux supports de cire au lieu d'un. Leur disposition permet l'étude de l'étirage des cires ou celle de l'élevage des larves d'ouvrières ou de reines.

De par les dimensions de leur socle, ces cagettes sont stables. De plus, leur faible hauteur permet d'en placer un grand nombre dans les étuves.

\section{Reçu pour publication en novembre 1965 .}

\section{SUMMARY}

\section{A NEW DESIGN FOR AN EXPERIMENTAL CAGE FOR KEEPING BEES IN CAPTIVITY}

The author describes a cage made of altuglas ( $\left.{ }^{(}\right)$, specially designed for keeping small groups of workers in captivity. The ease of handling and cleaning these cages is pointed out. The cages are specially adapted for studying the feeding habits of bees.

\section{RÉFÉRENCES BIBL,IOGRAPHIQUES}

Pain J. 196ı. Sur la phérormone des reines d'abeilles et ses effets physiologiques. Thèse Sci. Nat. Paris. $n^{\circ} 4526$

(1) See (1) p. 7I. 